Díaz, J.; Muñoz, D.; Muñoz, J. y Ibáñez, S.J. (2021) Design and Validation of an Observational Instrument for Final Actions in Padel. Revista Internacional de Medicina y Ciencias de la Actividad

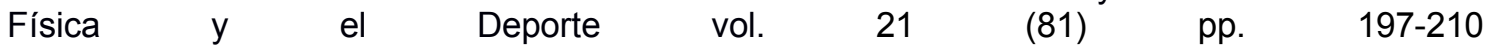
Http://cdeporte.rediris.es/revista/revista81/artdiseno1237.htm

DOI: https://doi.org/10.15366/rimcafd2021.81.013

\title{
ORIGINAL
}

\section{DISEÑO Y VALIDACIÓN DE UN INSTRUMENTO OBSERVACIONAL PARA ACCIONES FINALISTAS EN PÁDEL}

\section{DESIGN AND VALIDATION OF AN OBSERVATIONAL INSTRUMENT FOR FINAL ACTIONS IN PADEL}

\author{
Díaz, J. ${ }^{1} ;$ Muñoz, D. ${ }^{2}$; Muñoz, J.,4 e lbáñez, S.J. ${ }^{5}$ \\ ${ }^{1}$ Doctorando en Ciencias del Deporte (UEx). Facultad de Ciencias del Deporte. Universidad de \\ Extremadura (España) jediazg@alumnos.unex.es \\ 2 Profesor Titular de Universidad, Facultad de Ciencias del Deporte. Universidad de Extremadura \\ (España)diegomun@unex.es \\ 3 Profesor Colaborador de Universidad, Facultad de Ciencias del Deporte. Universidad de \\ Extremadura (España) suliwan@unex.es \\ ${ }^{4}$ Investigador asociado. Universidad Autónoma de Chile (Chile) jesus.munoz@uautonoma.cl \\ ${ }^{5}$ Catedrático de Universidad, Facultad de Ciencias del Deporte. Universidad de Extremadura \\ (España) sibanez@unex.es
}

Código UNESCO / UNESCO code: 5899 Educación Física y Deportiva / Physical Education and Sports.

Clasificación Consejo de Europa / Council of Europe classification: 17. Otras: Análisis del Rendimiento / Others: Performance Analysis.

Recibido 9 de abril de 2019 Received April 9, 2019

Aceptado 15 de septiembre de 2019 Accepted September 15, 2019

\section{AGRADECIMIENTOS:}

Este estudio ha sido subvencionado parcialmente por las Ayudas a Grupos de Investigación (GR18170) de la Junta de Extremadura (Consejería de Empleo, Empresa e Innovación), con una aportación de la Unión Europea de los Fondos Europeos de Desarrollo Regional.

\section{RESUMEN}

El pádel es uno de los deportes con mayor crecimiento en España, lo que se ve reflejado en un número cada vez mayor de investigaciones. El objetivo del presente trabajo fue diseñar un instrumento de observación que permita analizar las acciones finalistas en el pádel (IPAAFP) y, además, comprobar su validez y fiabilidad. Un grupo de jueces expertos $(n=10)$, evaluaron en una escala 1-10 la Adecuación y Redacción de 17 items que componen el instrumento. La validez 
de contenido fue evaluada con la $V$ de Aiken $(N=0,83)$ y la congruencia interna con el $\alpha$ de Cronbach $(0,838)$. Ningún ítem tuvo que ser eliminado, pero sobre la base de las valoraciones cualitativas de los expertos se realizaron modificaciones en la redacción de los items. Tras el proceso de validación del instrumento, se puede concluir que el IPAAFP es válido y fiable para el análisis de las acciones finalistas en el pádel.

PALABRAS CLAVE: Deportes de raqueta, Observación, Validez de contenido, Fiabilidad.

\section{ABSTRACT}

During the last years, padel has been one of the fastest growing sports in Spain, reflected in an increase of investigations about it. So, the aim of this study was to design an observation instrument that allows us analyze how points are concluded in padel (IPAAFP). Also, the instrument must be valid and reliable. A group of padel expert judges $(n=10)$ evaluated the Adequacy and Writing of 16 items on an 1-10 scale. Aiken's V coefficient and Cronbach's $\alpha$ were the statistical coefficients used in that process. Neither of the items had to be eliminated $(V=.83)$ and the value of internal reliability was .838 , but we had to make small adjustments in IPAAFP. Finally, we can conclude that the IPAAFP is a valid and reliable instrument for the analysis of the final actions in the padel.

KEY WORDS: Racket sports, Observation, Content Validity, Reliability.

\section{INTRODUCCIÓN}

Realizar trabajos científicos que aborden como temática de estudio al pádel se puede justificar desde dos puntos de vista: el impacto social que tiene dicho deporte y la falta de cantidad y variedad temática de publicaciones que indagan sobre el mismo (Díaz, García, Grijota, Muñoz \& Muñoz, 2015; Sánchez-Alcaraz, Courel-Ibañez \& Cañas, 2018). Las publicaciones existentes hasta el momento acerca del pádel están centradas en la descripción de variables cinemáticas y psicofisiológicas (Díaz, Grijota, Robles, Maynar \& Muñoz, 2017; Carbonell, Ferrándiz \& Pascual, 2017). Por el contrario, a nivel técnico-táctico, no existe un sistema que aporte información y ayude en competición a los jugadores. Sin embargo, científicamente está probada la eficacia de incluir feedback sobre el rendimiento de los deportistas (Anguera, 1990).

Las técnicas observacionales, cuyo carácter científico está avalado (Suen \& Ary, 1989; Riba, 1991; Bakeman \& Gottman, 1997), despiertan un gran interés entre los investigadores de deportes de raqueta. De esta forma, existen ejemplos en los deportes de red, como el tenis (Gorospe, Hernández, Anguera \& Martínez, 2005) y tenis de mesa (Pradas, Floria, González-Jurado, Carrasco \& Bataller, 2012), o deportes de red y muro, como el Squash (Catalán \& González, 2015). Al contrario, referido al pádel, existen pocas investigaciones que analicen la técnica y la táctica a través de una herramienta observacional (Courel-Ibañez \& 
Sánchez-Alcaráz, 2017). Cabe destacar a Fernández de Osso \& León (2017), que presentan una herramienta de registro de la frecuencia del primer/segundo servicio, así como los marcadores temporales y los golpeos empleados. Sin embargo, consideramos que sigue sin existir un instrumento que garantice el análisis del juego en relación con el rendimiento que se está produciendo, pudiendo así ayudar con una base científica a nuestros deportistas incluso durante el propio partido, ya que la normativa de juego en el pádel permite la existencia de un técnico que apoye a los jugadores durante la competición, a diferencia, por ejemplo, del tenis. Por ello, en este estudio se procederá al diseño de una hoja de observación con las características mencionadas.

El gran número de acciones técnico - tácticas así como la velocidad en el juego de los deportes de raqueta, dificulta el análisis del juego mediante la observación, por lo que puede resultar interesante seleccionar indicadores de rendimiento a observar en lugar de observar la totalidad del juego. Este aspecto está justificado desde dos perspectivas. Por un lado, la primera perspectiva reside en que desde el punto de vista técnico y de organización eficaz del entrenamiento de alta competición (Hong, Robinson, Chan, Clark \& Choi, 1996; Malagoli, Lobietti \& Merni, 2010), así como desde la transmisión eficaz de la información entre el entrenador y el deportista (Moreno, Santos, Ramos, Sanz, Fuentes \& Del Villar, 2002). En pádel, estos análisis aislados pueden hacer referencia, entre otros, al instante del saque o al golpeo finalista, por ejemplo. Además, dentro de estos análisis aislados, algunos autores como Hughes, Hughes \& Behan (2007) y Over \& O'Donoghue (2010) concluyen que existen determinadas cuestiones que no pueden faltar en un sistema de categorías para deportes de raqueta, tales como el recurso utilizado, la duración de los intercambios, la eficacia de los golpeos, el número del servicio y/o si los jugadores se encuentran al saque o al resto. La segunda cuestión a tener en cuenta es que la observación es un campo de investigación con una extensa trayectoria científica y una metodología definida de los procesos de validación y fiabilidad de los instrumentos (Thomas, Silverman \& Nelson, 2015), que debe ser respetada

Basándose en esto, la finalidad de la presente investigación es el diseño de un instrumento de observación que permita describir y analizar las acciones finalistas con las que se terminan los puntos en pádel (en adelante IPAAFP), así como demostrar la validez y la fiabilidad de este instrumento.

\section{MÉTODO}

Atendiendo a Ato, López \& Benavente (2013), este estudio se definió dentro de la metodología instrumental, pues su objetivo fue elaborar y validar un nuevo instrumento de observación, para evaluar las acciones que finalizan el punto en el pádel.

\subsection{MUESTRA}

La muestra seleccionada para el estudio estuvo conformada por un conjunto de jueces expertos que valoraron el contenido de los ítems del instrumento (Rodríguez, Gil \& García, 1996). La denominación de juez experto está sometida 
a los criterios establecidos para el estudio, y basados en los conocimientos del deporte en cuestión y del procedimiento científico y/o validación (Valles, 2003; Escobar \& Cuervo, 2008). Cada juez debió cumplir, al menos, el $80 \%$ de los criterios definidos a continuación, es decir, 4 de 5. i) Poseer el título de Doctor; ii) Ser o haber sido profesor universitario; iii) Poseer la titulación federativa Nivel II en un deporte de raqueta; iv) Un mínimo de 10 años de experiencia como entrenador deportes de raqueta; v) Tener publicaciones con temática orientadas a un deporte de raqueta.

Se identificaron 15 sujetos que reunían los criterios de inclusión, de los cuales 10 respondieron al cuestionario (66\%). En la tabla 1 se muestran los criterios de inclusión que cumplieron cada uno de los jueces expertos.

Tabla 1. Criterios que cumplían los jueces expertos

\begin{tabular}{lrrrrrrrrrr}
\hline & \multicolumn{10}{c}{ Jueces expertos } \\
\cline { 2 - 10 } Criterios & $\mathrm{J} 1$ & $\mathrm{~J} 2$ & $\mathrm{~J} 3$ & $\mathrm{~J} 4$ & $\mathrm{~J} 5$ & $\mathrm{~J} 6$ & $\mathrm{~J} 7$ & $\mathrm{~J} 8$ & $\mathrm{J9}$ & $\mathrm{J} 10$ \\
\hline $\mathrm{C} 1$ & $\mathrm{X}$ & $\mathrm{X}$ & $\mathrm{X}$ & $\mathrm{X}$ & $\mathrm{X}$ & $\mathrm{X}$ & $\mathrm{X}$ & $\mathrm{X}$ & $\mathrm{X}$ & $\mathrm{X}$ \\
$\mathrm{C} 2$ & $\mathrm{X}$ & $\mathrm{X}$ & $\mathrm{X}$ & $\mathrm{X}$ & & $\mathrm{X}$ & $\mathrm{X}$ & $\mathrm{X}$ & $\mathrm{X}$ & $\mathrm{X}$ \\
$\mathrm{C} 3$ & $\mathrm{X}$ & $\mathrm{X}$ & & $\mathrm{X}$ & $\mathrm{X}$ & $\mathrm{X}$ & $\mathrm{X}$ & $\mathrm{X}$ & $\mathrm{X}$ & $\mathrm{X}$ \\
$\mathrm{C} 4$ & $\mathrm{X}$ & $\mathrm{X}$ & $\mathrm{X}$ & $\mathrm{X}$ & $\mathrm{X}$ & $\mathrm{X}$ & $\mathrm{X}$ & & $\mathrm{X}$ & $\mathrm{X}$ \\
$\mathrm{C} 5$ & $\mathrm{X}$ & $\mathrm{X}$ & $\mathrm{X}$ & $\mathrm{X}$ & $\mathrm{X}$ & $\mathrm{X}$ & $\mathrm{X}$ & $\mathrm{X}$ & $\mathrm{X}$ & $\mathrm{X}$ \\
\hline
\end{tabular}

\subsection{Variables}

Se definieron las variables que conformaban el contenido del instrumento. Además, se identificaron las variables estadísticas para su validación (Escobar \& Cuervo, 2008). Finalmente, el instrumento incluyó 16 variables de contenido

Por otro lado, en el proceso de validación del instrumento, se tuvieron en cuenta dos variables:

A) Validez de contenido. Obtenida a través del estadístico $V$ de Aiken (Aiken, 1985), que define el grado de adecuación de cada uno de los constructos que conforman un objeto de estudio (Abad, Olea, Ponsoda \& García, 2011).

B) Congruencia interna. Calculada en el estudio mediante el $\alpha$ de Cronbach (Cronbach, 1990), que mide el grado de correlación entre los distintos ítems de una misma prueba (Abad et cols., 2011).

La elaboración del instrumento fue sometida al juicio de expertos, que debían puntuar en una Escala Likert de 1 a 10 los grados de Adecuación (pertinencia al objeto de estudio) y de Redacción (precisión y corrección), por separado, para cada uno de los ítems definidos. Además, cabía la posibilidad de realizar una valoración subjetiva de uno o varios ítems en el apartado de observaciones.

De esta forma, se obtuvieron una serie de variables cuantitativas y cualitativas, que posteriormente recibieron un tratamiento matemático, para poder elaborar 
un instrumento de observación válido y fiable para el análisis de las acciones finalistas en pádel.

\subsection{INSTRUMENTO}

A continuación, se presentaron las 16 variables de análisis que conformaron el instrumento final en base a su núcleo categorial y los diferentes rangos de apertura (Anguera, 1991):

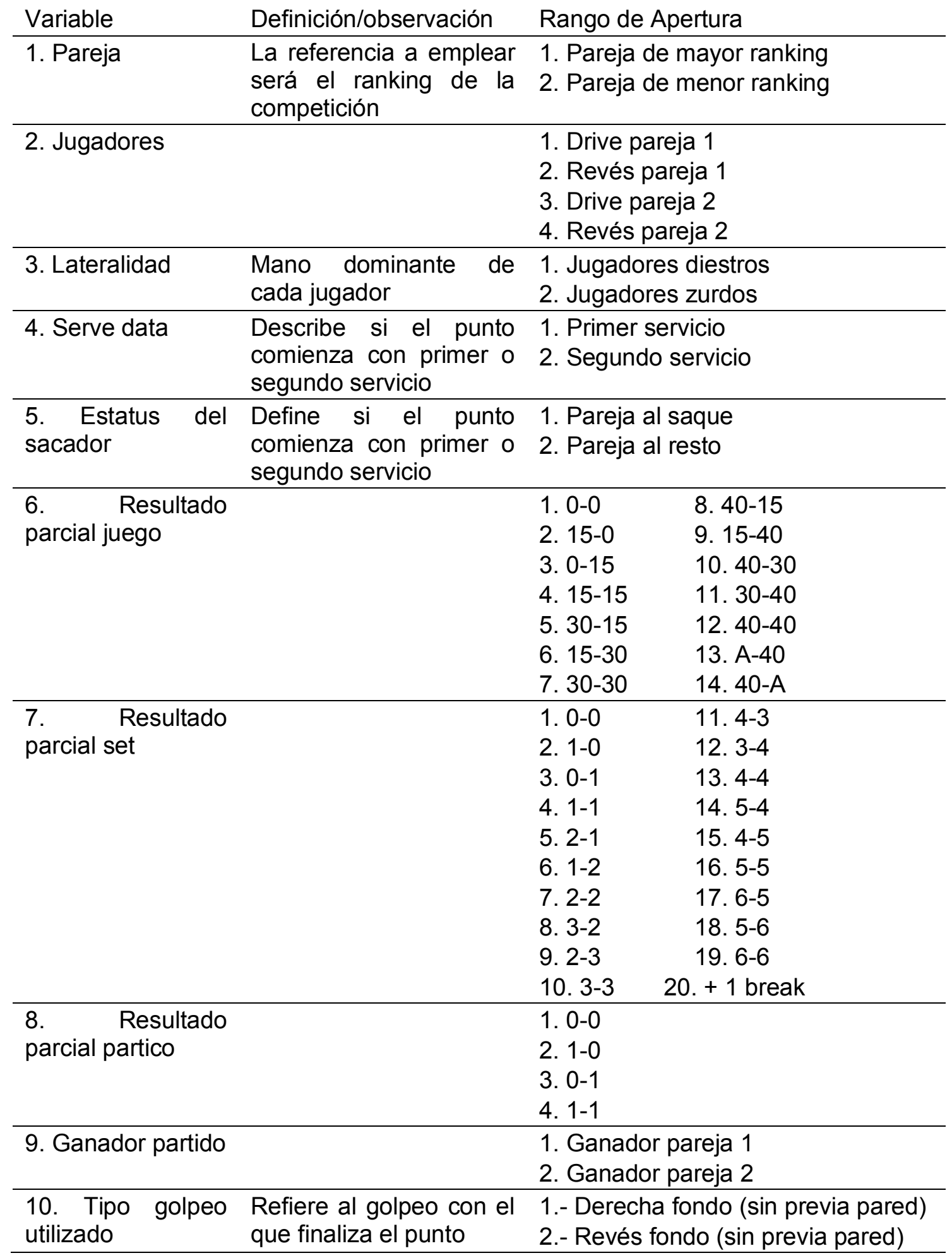




\begin{tabular}{|c|c|c|}
\hline & & \multirow[b]{2}{*}{$\begin{array}{l}\text { 3.- Derecha fondo-pared } \\
\text { 4.- Reves fondo-pared } \\
\text { 5.- Globo } \\
\text { 6.- Volea derecha } \\
\text { 7.- Volea de revés } \\
\text { 8.- Bandeja } \\
\text { 9.- Remate }\end{array}$} \\
\hline & & \\
\hline $\begin{array}{l}\text { 11. Zona golpeo } \\
\text { finalizador }\end{array}$ & $\begin{array}{l}\text { Zona desde la que el } \\
\text { jugador golpea la bola } \\
\text { que ginaliza el punto }\end{array}$ & \\
\hline $\begin{array}{l}\text { 12. Zona de envio } \\
\text { bola previa a la } \\
\text { finalización }\end{array}$ & $\begin{array}{l}\text { Zona desde la que el } \\
\text { jugador contrario envía } \\
\text { la bola al jugador que } \\
\text { finaliza el punto }\end{array}$ & Campograma \\
\hline $\begin{array}{l}\text { 13. Zona destino } \\
\text { golpeo finalizador }\end{array}$ & $\begin{array}{l}\text { Zona a la que el jugador } \\
\text { que finaliza el punto } \\
\text { envía esa bola final }\end{array}$ & Campograma \\
\hline 14. Eficacia & $\begin{array}{l}\text { Modo en que finaliza el } \\
\text { punto }\end{array}$ & $\begin{array}{l}\text { 1. Éxito. Remate x4. } \\
\text { 2. Éxito. Remate x3. } \\
\text { 3.- Éxito. Doble Bote. } \\
\text { 4.- Éxito. Contacto cuerpo. } \\
\text { 5.- Éxito. Provocar error forzado. } \\
\text { 6.-Éxito Otros. } \\
\text { 7.- Fracaso. Bola directa red. } \\
\text { 8.- Fracaso. Bola directa elementos } \\
\text { laterales o fondo. } \\
\text { 9.- Fracaso Otro }\end{array}$ \\
\hline \multirow[t]{3}{*}{ 15. Rallie } & Número de golpeos del & \\
\hline & $\begin{array}{l}\text { Intercambio previo a la } \\
\text { finalización del punto }\end{array}$ & $\begin{array}{l}2 . \quad \text { Rallie intermedio } \quad(8-16 \\
\text { golpeos) }\end{array}$ \\
\hline & & 3. Rallie largo (> 16 golpeos) \\
\hline
\end{tabular}

\subsection{ANÁLISIS DE DATOS}

La Validez de Contenido del IPAAFP se calculó mediante el coeficiente $V$ de Aiken (Aiken, 1985), que es uno de los más extendidos cuando se trata de valoraciones de grupo de expertos. El valor de este coeficiente oscila entre $0 \mathrm{y}$ 1 , donde 1 indica una concordancia perfecta entre los jueces respecto al contenido evaluado. Para su cálculo se utilizó el programa Visual Basic 6.0, que emplea la formula modificada de Peinfield \& Giacobbi (2004):

$$
V=\frac{\bar{X}-l}{k}
$$

$\dot{X}$ hace referencia a la media de las puntuaciones obtenidas por los jueces, I es el valor más bajo de la escala (1) y $K$ es su rango (10-1=9). El programa proporciona los valores de la $V$ de Aiken y sus intervalos de confianza al 90, 95 
y 99\%. Se tuvieron en cuenta los valores del 95\% (Merino y Livia, 2009), recomendado para las investigaciones en Ciencias Sociales.

Una vez calculado la $V$ de Aiken, el siguiente paso fue calcular el valor crítico de dicha variable, según el cual un ítem o variable debe ser eliminado, modificado o aceptado. Atendiendo a las características de la muestra y del IPAAFP hubo que utilizar el "Large-Sample Test for the Mean of V" o teorema del límite central para grandes muestras:

$$
V=\frac{z}{.2 \sqrt{3 \operatorname{mn}(c+1) /(c-1)}}+.5
$$

En esta fórmula, $Z$ es el valor significativo de la validez de contenido $(Z=1.645$ en .95), $\mathrm{m}$ es el número de ítems del instrumento $(m=16)$ y $\mathrm{n}$ corresponde al número de jueces expertos que participaron en la recogida de datos $(n=10)$, siendo $c$ las diferentes posibilidades de respuesta de los mismos $(c=10)$. Según estos cálculos, el valor para el IPAAFP en base a sus características es de $V=0.83$ para rechazar la hipótesis nula. Es decir, se deberían descartar todos los ítems que se encuentren por debajo de esos valores para la $V$ de Aiken. Además, se atendió a todas las sugerencias de los expertos, para la modificación y mejora de los ítems del instrumento.

Por último, para analizar la congruencia interna, se utilizó el $\alpha$ de Cronbach y el programa estadístico SPSS 19.0 para realizar los cálculos. Siguiendo a Field (2009) y Polit \& Hungler (2000) se estableció en 0.70 el valor a partir del cual considerarlos fiables.

\section{RESULTADOS}

A continuación, se exponen los resultados obtenidos en los análisis de validez y fiabilidad del IPAAFP:

En la tabla 2 aparecen los resultados de la $V$ de Aiken para la Validez de la Adecuación y Redacción de cada una de las categorías. 
Tabla 2. Valores $\mathrm{V}$ de Aiken para la Adecuación y Redacción del IPAAFP

\begin{tabular}{lrrrrrr}
\hline & \multicolumn{3}{c}{ Adecuación } & \multicolumn{3}{c}{ Redacción } \\
\hline \multicolumn{1}{c}{ Variable } & Media & $\begin{array}{c}\text { V de } \\
\text { Aiken }\end{array}$ & $\mathbf{9 5 \%}$ & Media & $\begin{array}{c}\text { V de } \\
\text { Aiken }\end{array}$ & \multicolumn{1}{c}{$\mathbf{9 5 \%}$} \\
\hline Parejas & 9.2 & 0.88 & $\mathbf{0 . 8 - 0 . 9 3}$ & 9.1 & 0.88 & $\mathbf{0 . 8 - 0 . 9 3}$ \\
Jugador & 10 & 1 & $\mathbf{0 . 9 5 - 1}$ & 10 & 1 & $\mathbf{0 . 9 5 - 1}$ \\
Lateralidad & 10 & 1 & $\mathbf{0 . 9 5 - 1}$ & 10 & 1 & $\mathbf{0 . 9 5 - 1}$ \\
Serve Data & 9.4 & 0.88 & $\mathbf{0 . 8 - 0 . 9 3}$ & 9.3 & 0.88 & $\mathbf{0 . 8 - 0 . 9 3}$ \\
Estatus del Sacador & 10 & 1 & $\mathbf{0 . 9 5 - 1}$ & 10 & 1 & $\mathbf{0 , 9 5 - 1}$ \\
Resultado Parcial Juego & 9.4 & 0.88 & $\mathbf{0 . 8 - 0 . 9 3}$ & 9.2 & 0.88 & $\mathbf{0 . 8 - 0 . 9 3}$ \\
Resultado Parcial Set & 9.4 & 0.88 & $\mathbf{0 . 8 - 0 . 9 3}$ & 9.4 & 0.88 & $\mathbf{0 . 8 - 0 . 9 3}$ \\
Resultado Parcial Partido & 10 & 1 & $\mathbf{0 . 9 5 - 1}$ & 10 & 1 & $\mathbf{0 . 9 5 - 1}$ \\
Ganador del Partido & 10 & 1 & $\mathbf{0 . 9 5 - 1}$ & 10 & 1 & $\mathbf{0 . 9 5 - 1}$ \\
Tipo Golpeo Utilizado & 9.1 & 0.88 & $\mathbf{0 . 8 - 0 . 9 3}$ & 9.1 & 0.88 & $\mathbf{0 . 8 - 0 . 9 3}$ \\
Zona Golpeo Finalizador & 9.1 & 0.88 & $\mathbf{0 . 8 - 0 . 9 3}$ & 9.1 & 0.88 & $\mathbf{0 . 8 - 0 . 9 3}$ \\
Zona Envín Bola Previa & 9.1 & 0.88 & $\mathbf{0 . 8 - 0 . 9 3}$ & 9.2 & 0.88 & $\mathbf{0 . 8 - 0 . 9 3}$ \\
Zona Destino & 9.1 & 0.88 & $\mathbf{0 . 8 - 0 . 9 3}$ & 9.1 & 0.88 & $\mathbf{0 . 8 - 0 . 9 3}$ \\
Campograma & 9.2 & 0.88 & $\mathbf{0 . 8 - 0 . 9 3}$ & 9.2 & 0.88 & $\mathbf{0 . 8 - 0 . 9 3}$ \\
Eficacia & 9.1 & 0.88 & $\mathbf{0 . 8 - 0 . 9 3}$ & 9.2 & 0.88 & $\mathbf{0 . 8 - 0 . 9 3}$ \\
Rallie & 9.9 & 0.88 & $\mathbf{0 . 8 - 0 . 9 3}$ & 9.9 & 0.88 & $\mathbf{0 . 8 - 0 . 9 3}$ \\
& & & & & & \\
\hline
\end{tabular}

Como se puede observar, todos los ítems superan los 0.83 que se establecieron como valor crítico para la $V$ de Aiken.

En la tabla 3 se exponen un resumen de las valoraciones cualitativas que los expertos hicieron a nuestro instrumento.

Tabla 3. Valoraciones cualitativas expertos.

\begin{tabular}{lcl}
\hline Item & Experto & Observaciones \\
\hline 14 & 6 & "Podrían definirse más zonas" \\
14 & 9 & "Correcto, mejorarlo implica difícil observación" \\
14 & 2 & "En expertos se requieren más zonas" \\
7 & 10 & "Indicar breaks producidos" \\
7 & 9 & "Breaks" \\
10 & 9 & "Indicar altura golpeos" \\
10 & 9 & "Otras posibilidades clasificación" \\
\hline
\end{tabular}

Por último, en la tabla 4 se exponen los valores de congruencia interna, a través del $\alpha$ de Cronbach, tanto de la adecuación y la redacción como del instrumento completo.

Tabla 4. Valores Alfa de Cronbach para el IPAAFP.

\begin{tabular}{cccc}
\hline & Adecuación & Redacción & Instrumento \\
\hline$\alpha$ & 0.73 & 0.82 & 0.838 \\
$n$ & 16 & 16 & 32 \\
\hline
\end{tabular}


En este caso, se obtuvieron valores de fiabilidad situados por encima de los 0.70 puntos que establecían Field (2009) y Polit \& Hungler (2000), para la validación del instrumento.

\section{DISCUSIÓN}

El propósito de este estudio fue elaborar y validar una hoja de observación para el análisis de los golpeos finalistas en el pádel, con garantías de validez y fiabilidad.

Si bien la literatura de otros deportes de raqueta muestra que existen múltiples aplicaciones de los instrumentos de observación en los mismos, en pádel son reducidas las intervenciones que se han realizado para esta línea de investigación. Uno de los pocos ejemplos a destacar, es el trabajo de Fernández de Osso \& León (2017), que registran una serie de variables cuantitativas no relacionadas con el resultado ni la eficacia de las decisiones de los deportistas. Este trabajo busca solucionar problemas tácticos en el pádel, por lo que puede considerarse pionero en el deporte en cuestión, ya que incluye un análisis del Serve Data y del tipo de golpeo como indicadores del rendimiento deportivo. El IPAAFP, por su parte, es también uno de los primeros trabajos de investigación que busca relacionar la metodología observacional con el deporte del pádel de forma específica. Sin embargo, a diferencia del anterior, el IPAAFP ha sido diseñado con el objetivo de encontrar relación entre las variables tácticas definidas por los expertos y el resultado de las mismas en valores de eficacia, que en un futuro puede concluir con la definición, , de indicadores de rendimiento específicos del deporte del pádel.

Respecto a la definición de las variables, Fernandez de Osso \& León (2017) establecían el Serve Data y el tipo de golpeo como variables de estudio técnicotácticas, así como otras citadas en la bibliografía por O’Donoghue \& Ingram (2001), O’Donogue \& Liddle (1998) o Johnson \& McHugh (2006). Estos autores concluían que había una serie de variables relevantes de estudio en los deportes de raqueta. Sus análisis, demostraban la relación con los indicadores de rendimiento. Los trabajos de estos investigadores se centraban principalmente en el tenis, pero algunas variables como el rallie o la eficacia del golpeo son aplicables a otros deportes de raqueta, y fueron de gran ayuda para enriquecer al instrumento que se presenta en este trabajo. En este sentido, algunos trabajos que no se han centrado en el diseño de instrumentos pero si han investigado sobre la táctica en pádel (Muñoz, Sánchez-Alcaraz, Courel, Díaz, Julián \& Muñoz, 2017; Courel \& Sanchez Alcaraz, 2017) han servido de gran ayuda para la definición del instrumento.

Finalmente, el IPAAFP define hasta 16 variables de rendimiento, dando lugar a una caracterización muy completa de la situación a analizar, ya que se describen a los contrincantes (jugador, pareja y lateralidad), se establecen los resultados finales y parciales, y se describe la situación de definición (golpeo, zonas de juego y eficacia). En concreto, referido a las zonas de golpeo, la elaboración de un campograma es otra de las aportaciones que se presentan en este trabajo. No ha sido encontrado en la bibliografía consultada otro estudio con estas características. Si bien este campograma podría estar dividido en más partes, 
sobre todo en la zona de ataque, el establecimiento de las líneas del campo como referencia tiene como objetivo facilitar la labor del observador y garantizar la fiabilidad de los datos que le llegan al entrenador o investigador.

Respecto a la elaboración de las categorías del IPAAFP, Fernández de Osso \& Leon, (2017), establecen dos situaciones de juego de análisis: el saque y el juego posterior en su conjunto global, contabilizado por todos los golpeos que se producen. En otros deportes, como el voleibol (Moreno et. Cols.,2002) y o los deportes de raqueta (Hong et. Cols, 1996), definen que la focalización de la atención en una situación concreta del juego puede resultar una estrategia eficaz en la observación y la transmisión de información al deportista. En este sentido, el IPAAFP no es solo uno de los primeros instrumentos centrados exclusivamente en el deporte del pádel, además se centra en un solo aspecto del juego, en este caso uno tan determinante como el golpeo finalizador. El IPAAFP puede convertirse en una ayuda para competición y entrenamiento en pádel, y en este sentido los autores anteriormente mencionados explican que la especialización es una característica fundamental de la tecnificación deportiva.

Posteriormente, una vez definidas las variables del estudio, era necesario demostrar la validez del instrumento de observación. Si bien son varias las metodologías definidas para registrar la validez de un instrumento de estas características, para el presente trabajo se ha optado por el coeficiente $V$ de Aiken, que es uno de los más utilizados para valorar grupos de expertos, lo que permite contrastar los resultados con numerosa bibliografía existente. Aiken (1985), define una fórmula, anteriormente expuesta, para calcular su coeficiente, y también describe que en función de los números de jueces implicados y posibilidades de respuesta el valor crítico para la aceptación de los ítems debe variar, de tal forma que mientras mayor sea el tamaño de la muestra de expertos menor serán las exigencias en este valor, pues se entiende que el consenso es implícito. Cuervo \& Escobar (2008) citan de forma complementaria que si el $80 \%$ de los jueces están de acuerdo en una cuestión esta debe ser aprobada, pero Aiken (1985) detalla en profundidad su procedimiento: todo valor del coeficiente por encima del valor crítico para la $V$ de Aiken debe ser aceptado, y este valor es específico para cada estudio.

En este sentido, el coeficiente $V$ de Aiken ha sido empleado en otros trabajos similares de reciente publicación. García-Santos \& Ibáñez (2016) obtuvieron un valor crítico de $\mathrm{V}=0.69$ en la validación de su instrumento sobre el arbitraje en baloncesto. Conejero, Claver, Fernández, González \& Moreno (2017) obtuvieron un valor de $\mathrm{V}=0.67$ en un estudio sobre la toma de decisiones en voleibol, con similitudes respecto al presente trabajo en cuanto a la selección de una acción concreta del juego a observar, en este caso la recepción en voleibol. También en esta línea, García-Martín, Antúnez e Ibáñez (2016) presentan valores de $\mathrm{V}=0.70$ en trabajo de proceso formativo en jugadores expertos de baloncesto. Como se puede observar, en todos estos trabajos anteriormente presentados los valores de la $V$ de Aiken superan el 0.70 , pero ninguno llega a ser tan alto como el .83 del presente estudio, por lo que se expone uno de los trabajos más exigentes de los realizados hasta el momento. El alto nivel de exigencia en el IPAAFP puede ser debido a su número ítems, expertos y rango de respuesta, ya que como se explicaba anteriormente, los divisores de la fórmula de Aiken (1985) 
dependen de todos ellos. Aun así, ninguno de los ítems del presente trabajo debió ser eliminado. Probablemente, el motivo por el cual se obtuvieron valores tan altos de puntuación sea que existe un consenso global entre los expertos a la hora de conocer las necesidades técnicas y tácticas de pádel. Para las modificaciones necesarias de los ítems se tuvieron en cuenta las valoraciones cualitativas de los expertos, realizadas en el apartado de observación de las fichas que les fueron proporcionadas.

Por último, para la fiabilidad del instrumento fue empleado el coeficiente Alfa de Cronbach (Cronbach, 1990). La elección de este coeficiente fue su contrastada utilización en investigaciones de ciencias sociales, que tienen características similares a la del presente estudio. Este estadístico permite obtener la fiabilidad tanto para el instrumento en su totalidad como para la Redacción y Adecuación por separado. Field (2009) y Polit \& Hungler (2000), consideran que todos los valores por encima de 0.70 define el instrumento como fiable a nivel interno.Los valores obtenidos de este coeficiente en el presente estudio fue de 0.83. Otros trabajos, como el de González-Espinosa, Feu, García, Antúnez y García-Santos (2017), alcanzaron un valor superior de 0.960 para su comparación entre diferentes metodologías de enseñanza del baloncesto. Sin embargo, en el trabajo de valoración de los árbitros en baloncesto de García Santos e Ibáñez (2016), obtuvieron un valor menor, en concreto de 0.712. Como se puede comprobar, el presente estudio obtiene un valor intermedio respecto a los trabajos previos, en la congruencia interna, que por tanto lo define como un instrumento fiable, al superar muy por encima los 0.70 establecidos como referencia por Field (2009) y Polit \& Hungler (2000) para este estadístico. Estos valores obtenidos pueden ser explicados al tratarse de un conjunto de expertos con unas ideas muy concretas sobre las necesidades del deporte en cuestión, y ser un grupo de personas definidas por unas características intencionales, concretas y deliberadas por los investigadores del trabajo.

\section{CONCLUSIONES}

EI IPAAFP es un instrumento de análisis del pádel novedoso y completo, que incluye hasta 16 variables de análisis, entre ellas un campograma.

EI IPAAFP es un instrumento válido y de probada fiabilidad para evaluar las acciones finalistas en el pádel.

\section{REFERENCIAS BIBLIOGRÁFICAS}

Abad, F. J., Olea, J., Ponsoda, V., and García, C. (2011). Medición en ciencias sociales y de la salud (Measurement in Social and Educational Sciences). Madrid. Síntesis.

Aiken, L. R. (1985). Three coefficients for analyzing the reliability and validity of ratings. Educational and Psychological Measurement, 45(1), 131142. https://doi.org/10.1177/0013164485451012

Anguera, M.T. (1990). Metodología observacional. En J. Arnau, M.T. Anguera y J. Gómez, Metodología de la investigación en Ciencias del 
Comportamiento (pp. 125-236). Murcia: Secretariado de Publicaciones de la Universidad de Murcia.

Anguera, M. T. (1991). Metodología observacional en la investigación psicológica. Barcelona: PPU.

Ato, M., Lopez, J. and Benavente, A. Un sistema de clasificación de los diseños de investigación en psicología. Anales de psicología, 29, 1038-1059. https://doi.org/10.6018/analesps.29.3.178511

Bakeman, R. and Gottman, J.M. (1997). Observing behavior. An introduction to sequential analysis, 2nd ed. Cambridge: Cambridge University Press.

Carbonell, J.A., Ferrándiz, J., and Pascual, N. (2017). Análisis de la frecuencia cardiaca en el pádel femenino amateur. Retos, Nuevas tendencias en Educación Física, Deporte y Recreación, 32, 204-207. https://doi.org/10.47197/retos.v0i32.56040

Catalán, M. and González, S. (2015). Validación de un instrumento de evaluación en deportes de red-muro: squash. Retos, 27, 73-80. https://doi.org/10.47197/retos.v0i27.34351

Conejero, M., Claver, F.; Fernández-Echeverría, C. González-Silva, J., and Moreno-Arroyo, M.P.; (2017). Diseño y validación de un instrumento de observación para valorar la toma de decisiones en la acción de recepción en voleibol. Cultura, Ciencia y Deporte, 67-75.

Courel-Ibáñez, J., y Sánchez-Alcaraz, B.J. (2017). Efecto de las variables situacionales sobre la duración y eficacia de los puntos en jugadores de pádel de élite. Apunts, Educación Física y Deportes, 127, 68-74. https://doi.org/10.5672/apunts.2014-0983.es.(2017/1).127.07 https://doi.org/10.5672/apunts.2014-0983.cat.(2017/1).127.07

Courel, J. and Sanchez-Alcaraz, BJ. (2017). Efecto de las variables situacionales sobre los puntos en jugadores de pádel de élite. Apunts. Educación Física y Deportes; 127(1): 68-74. https://doi.org/10.5672/apunts.2014-0983.es.(2017/1).127.07

Cronbach, L.J. (1990). Essentials of psychological testing (5th Ed.). New York: Harper \& Row.

Díaz, J. García, A. Grijota, F., Muñoz, J., and Muñoz, D. (2015). Incidencia de la importancia del punto sobre variables temporales en padel de primera categoría regional. Revista Andaluza Medicina del Deporte, 8(4);184-189.

https://doi.org/10.1016/j.ramd.2015.08.009

Díaz, J., Grijota, FJ., Robles, MC., Maynar, M., and Muñoz, D. (2017). Estudio de la carga interna en padel amateur mediante la frecuencia cardiaca. Apunts. Educación Física y Deportes, 127; 79-85.

Escobar, J. and Cuervo, A. (2008). Validez de contenido y juicio de expertos: una aproximación a su utilización. Avances en Medición, 6, 27-36.

Fernández de Osso, A. and León, JA. (2017) Herramienta de evaluación técnico-táctica en padel. Revista internacional de medicina y ciencias de la actividad física y el deporte, 17(68), 693-714. https://doi.org/10.15366/rimcafd2017.88.008

Field, A. (2009). Discovering statistics using SPSS. Los Angeles: Sage publications. 
García-Martín, A., Antúnez, A., and Ibáñez, S. J. (2016). Análisis del proceso formativo en jugadores expertos: validación de instrumento/Analysis of expert players' training process: validation of tools. Revista Internacional de Medicina y Ciencias de la Actividad Física y del Deporte, (61). https://doi.org/10.6018/264601

García-Santos, D. and Ibáñez, S. J. (2016). Diseño y validación de un instrumento de observación para la valoración de un árbitro de baloncesto (IOVAB). SPORT TK-Revista EuroAmericana de Ciencias del Deporte, 5(2), 15-26.

González Espinosa, S., Feu Molina, S., García Rubio, J., Antúnez, A., and García Santos, D. (2017). Diferencias en el aprendizaje según el método de enseñanza-aprendizaje en el baloncesto. Revista de psicología del deporte, 26(3), 0065-70.

Gorospe, G., Hernández, A., Anguera, MT. y Martínez, R. (2005). Desarrollo y optimización de una herramienta observacional en el tenis de individuales. Psicothema 17(1), 123-127.

Hong, Y., Robinson, P., Chan, W., Clark, C., and Choi, T. . (1996). Notational analysis on game strategy used by the world's top male squash players in international competition. Australian Journal Of Science And Medicine In Sport, 28(1), 18-23

Hughes, M., Hughes, M.T., and Behan, H. (2007). The Evolution of Computerised Notational Analysis Through the Example of Racket Sports. International Journal of Sports Science and Engineering, 1(1), 3-28. https://doi.org/10.21797/ksme.2008.10.3.001

Johnson, C D. and McHugh, M P. (2006). Performance demands of professional male tennis players. British Journal of Sports Medicine, 40, 696-699. http://doi.org/10.1136/bjsm.2005.021253

Malagoli, I., Lobietti, R., and Merni, F. (2010). Footwork in Relationship with Strokes and Efficacy during the 29th Olympic Games Table Tennis Final International Journal of Table Tennis Sciences, 6, 60-63.

Merino, C., and Livia, J. (2009). Intervalos de confianza asimétricos para el índice la validez de contenido: Un programa visual basic para la $\mathrm{V}$ de Aiken. Anales de Psicología, 25(1), 169-171.

Moreno, MP., Santos, JA., Ramos, L., Sanz, D., Fuentes, JP y Del Villar, F. (2002). Revista Motricidad 9, 119-140.

Muñoz, D., Sanchez-Alcaraz, BJ., Courel, J., Díaz, J., Julian, A. and Muñoz, J. (2017). Diferencias en las acciones de subida a la red en padel entre jugadores profesional y avanzados. Journal of Sport and Health Research; 9(2): 223-232.

O'Donoghue, P. and Ingram, B. (2001). A notational analysis of elite tennis strategy. Journal of Sports Sciences, 19(2), 107-115. https://doi.org/10.1080/026404101300036299

O'Donoghue, P. and Liddle, S.D. (1998). A Match Analysis of Elite Tennis Strategy for Ladies Singles on Clay and Grass Surface. In A. Lees, I. Maynard, M. D. Hughes \& T. Reilly (Eds.), Science and Racket Sports II (pp. 247-253). London: E \& FN Spon.

Over, S. and O'Donoghue, P. (2010). Analysis of Strategy and Tactics in Tennis. ITF Coaching and Sport Science Review, 18(50), 15 - 16. 
Penfield, R. and Giacobbi, P. (2004). Applying a score confidence interval to Aiken's item content-relevance index. Measurement in Physical Education and Exercise Science, 8(4), 213-225. https://doi.org/10.1207/s15327841mpee0804 3

Pradas, F., Floria, P., González-Jurado, J.A., Carrasco, L., and Bataller, V. (2012). Desarrollo de una herramienta de observación para el análisis de la modalidad individual del tenis de mesa. Journal of Sport and Health Research 4(3), 255-268.

Polit, D. and Hungler, B. (2000). Investigación científica en Ciencias de la Salud (6a ed.). México: McGraw-Hill.

Riba, C. (1991). El método observacional. Decisiones básicas y objetivos. En M.T. Anguera (Ed.), Metodología observacional en la investigación psicológica (pp. 29-114). Barcelona: P.P.U., vol. I.

Rodríguez, G., Gil, J. and García, E. (1996). Métodos de investigación cualitativa. Málaga: Aljibe.

Sanchez-Alcaraz, B.J., Courel-Ibañez, J. and Cañas, J. (2018). Estructura temporal, movimientos en pista y acciones de juego en padel. Retos: nuevas tendencias en educación física, deporte y recreación, 33, 308312.

Suen, H.K. and Ary, D. (1989). Analyzing quantitative behavioral observation data. Hilsdalle, N.J.: Lawrence Erlbaum Associates.

Thomas, J. R., Silverman, S., and Nelson, J. (2015). Research methods in physical activity, 7E. Human kinetics.

Valles, M. (2003). Técnicas cualitativas de investigación social: Reflexión metodológica y práctica profesional. Madrid: Editorial Síntesis.

Número de citas totales/Total references: $40(100 \%)$

Número de citas propias de la revista/Journal's own references: 2 $(5.00 \%)$

Rev.int.med.cienc.act.fís.deporte - vol. 21 - número 81 - ISSN: 1577-0354 